\title{
Kikuchi-Fujimoto disease developing in systemic lupus erythematosus in a male patient - A challenging case report and literature review
}

\author{
Hemant Kumar Sharma ${ }^{1, A-F \oplus}$ \\ ${ }^{1}$ Department of Pathology, Siliguri, India \\ A - Research concept and design, B - Collection and/or assembly of data, C - Data analysis and interpretation, \\ $D$ - Writing the article, E - Critical revision of the article, F-Final approval of article
}

Hemant Kumar Sharma. Kikuchi-Fujimoto disease developing in systemic lupus erythematosus in a male patient: A challenging case report and Literature review. J Pre-Clin Clin Res. 2020; 14(2): 29-32. doi: 10.26444/jpccr/123949

\begin{abstract}
Kikuchi-Fujimoto disease (KFD) is an infrequent benign self-limiting disease predominantly affecting young Asian women and has an excellent prognosis. Diagnosis of KFD is confirmed by characteristic immunohistology of lymph node biopsy revealing histiocytic necrotizing lymphadenitis with abundant CD 68+ plasmacytoid monocytes in the paracortex and karyorrhectic debris. SLE, in particular, has been reported to precede, occur simultaneously, or appear after KFD. Treatment modalities and clinical outcomes of both KFD and SLE are vastly different. Hence, it is imperative to make timely correct diagnoses of SLE and treat early to avoid multi-organ failure and potentially fatal outcomes. The case report is presented of a challenging case of a 26-year-old Asian male who was initially diagnosed with KFD, and subsequently met the diagnostic criteria for SLE. He was treated for SLE for 6 months and showed dramatic improvement symptomatically without any further recurrence.
\end{abstract}

\section{Key words}

systemic lupus erythematosus, lymphadenopathy, kikuchi-fujimoto, histiocytic necrotizing lymphadenitis, autoimmunity

\section{INTRODUCTION}

Kikuchi-Fujimoto disease (KFD) is an infrequent, benign, self-limiting disease, probably of viral or autoimmune pathogenesis, and predominantly affects young Asian females (Male-Female ratio 1:4) [1, 2, 3]. Diagnosis of KFD is confirmed by characteristic immunohistology of lymph node biopsy revealing histiocytic necrotizing lymphadenitis, with abundant CD68+ plasmacytoid monocytes in the paracortex and karyorrhetic debris without granulocytic infiltrate [2]. Sporadic cases of KFD patients having other associated autoimmune diseases - Adult-Onset Still's disease, Polymyositis, Bilateral uveitis, and Systemic juvenile idiopathic arthritis, and most significantly, SLE has also been reported [3, 4]. SLE can occur before, co-exist, or appear after the diagnosis of KFD [2,4]. According to a literature review by Baenas et al., KFD has been reported to precede (30\%), occur simultaneously (47\%), or appear after the clinical appearance of SLE (23\%) [1]. Differentiation between KFD and SLE is pertinent due to significant differences in treatment protocols and clinical outcomes. The case report is presented to highlight the challenging case of a 26-yearold Asian male who was initially diagnosed with KFD, and subsequently met the diagnostic criteria for SLE.

\footnotetext{
Address for correspondence: Hemant Kumar Sharma, Department of Pathology, Siliguri, India

E-mail: hemant1303@gmail.com

Received: 05.05.2020; accepted: 15.06.2020; first published: 24.06.2020
}

\section{CASE REPORT}

A 26-year-old male patient reported to the hospital with complaints of intermittent low-grade fever for 3 months, together with weight loss of more than $20 \%$ of body weight $(65 \mathrm{Kg}$ to $50 \mathrm{Kg}$ ). Subsequently, he developed polyarthralgia involving both upper and lower limbs, along with alopecia. He did not give any history of cough, sweating, anorexia, contact with tuberculosis patient, and drug intake. No medically relevant past or family history could be elucidated. No focal symptoms or history of skin lesions, oral ulcers, proximal muscle weakness, joint swelling, photosensitivity, digital ulcers, or Raynaud's phenomenon. He was admitted with a provisional diagnosis of fever (investigation) and further work-up. Initial tests showed normocytic normochromic anaemia, relative lymphopenia, thrombocytopenia, raised erythrocyte sedimentation rate (ESR), elevated lactate dehydrogenase (LDH), and elevated hepatic enzymes (Tab. 1). Blood cultures, urine cultures, and a serologic workup for bacterial or viral infection were negative. Posteroanterior and lateral radiographs of the chest were normal. Computerized axial tomography scan imaging of chest showed a few small, palpable, non-matted lymph nodes in the left axillary region. The largest lymph node measuring about $1.0 \mathrm{~cm} \times 1.0 \mathrm{~cm}$, along with bilateral minimal pleural effusion and bilateral basal consolidation.

The patient was subjected to ultrasound-guided fine needle aspiration cytology (FNAC) of the left axillary lymph node which revealed granulomatous inflammation without any evidence of malignancy. However, Ziel-Neilson staining of smears did not show any acid-fast bacilli. Based on his symptomatology, lymph node cytology findings, and high prevalence in India, he was suspected of having TB, and 
Table 1. Initial laboratory investigations

\begin{tabular}{|c|c|}
\hline Parameter & Results (Normal range) \\
\hline Haemoglobin & $10.1 \mathrm{~g} / \mathrm{dl}(13-17 \mathrm{~g} / \mathrm{dl})$ \\
\hline Total leucocyte count & $78.00 /$ cu mm (4,000-11,000/cu mm) \\
\hline Differential leucocyte count & $\begin{array}{l}\text { Polymorphs - } 80 \% \text {, Lymphocytes - } \\
12 \% \text {, Monocytes - 3\%, Eosinophils } \\
-5 \% \text {, }\end{array}$ \\
\hline Platelets & $\begin{array}{l}90,000 / \text { cu mm }(1,50,000-4,50,000 / \\
\text { cu mm }\end{array}$ \\
\hline $\begin{array}{l}\text { Erythrocyte sedimentation rate }(\mathrm{mm} / \mathrm{h}) \\
\text { measured by } 1 \text { st hour by Westergreen } \\
\text { method }\end{array}$ & $100 \mathrm{~mm}$ fall $(<20$ fall) \\
\hline Peripheral smear & $\begin{array}{l}\text { RBC predominantly normocytic } \\
\text { normochromic, } \\
\text { no atypical cells seen }\end{array}$ \\
\hline Urinanalysis & Albumin trace, no RBC \\
\hline Mantoux test & $\begin{array}{l}8 \mathrm{~mm} \text { induration after } 72 \text { hours } \\
\text { (Positive test defined as }>15 \mathrm{~mm} \\
\text { induration) }\end{array}$ \\
\hline HIV Serology & Negative \\
\hline VDRL & Negative \\
\hline Lactate dehyrdogenase & 650 (80-22 units/L) \\
\hline Liver function tests & $\begin{array}{l}\text { Alanine aminotransferase }-53 \mathrm{U} / \mathrm{L} \\
(5-40 \text { units/L) } \\
\text { Aspartate aminotransferase }-65 \mathrm{U} / \mathrm{L} \\
(5-40 \text { units/L) }\end{array}$ \\
\hline Renal function tests & Within normal limits \\
\hline
\end{tabular}

empirically started on Anti-TB therapy (ATT). However, the patient did not show any response to ATT and continued to remain febrile. Bone marrow aspirate and biopsy were attempted to rule out any infective cause or haematolymphoid malignancy, but they showed only cellular reactive bone marrow. In an attempt to reach a diagnosis, left axillary lymph node biopsy was performed and returned in $10 \%$ formation for histopathological examination. Gross exanimation of the lymph node did not reveal any abnormality. However, on microscopic examination, it showed partial effacement of architecture, extensive area of coagulative necrosis, massive apoptosis with an accumulation of karyorrhectic debris and histiocytes, and absence of granulocytes and eosinophils. No haematoxylin body was identified, nor were granuloma or malignancy seen.

Based on the histopathology o the lymph node, the diagnosis of Histiocytic necrotizing lymphadenitis, along with a differential diagnosis of Lupus adenitis, was offered (Fig. 1-2). Subsequently, the patient's rheumatology profile was performed which revealed positive anti-nuclear antibody (ANA) in a speckled pattern, positive anti-ds DNA, anti-Sm antibodies, and low C3 \& C4 complement levels (Tab. 2). As the results of rheumatology work-up along with pleural effusion and haematologic abnormalities met minimum diagnostic criteria of SLE laid out by American College of Rheumatology (ACR), Diagnosis of SLE was confirmed. The patient was started on Oral Steroids in tapering dosage (1mg/kg bodyweight dosage) and supportive therapy. Within 48 hours, his fever subsided and he started feeling better. He was given Tablet Prednisolone $7.5 \mathrm{mg}$ in tapering dosage over 2 months and Tablet Hydroxychloroquine $400 \mathrm{mg}$ daily. On review after 6 months, he had regained $10 \mathrm{~kg}$ weight, remained afebrile and his laboratory investigations showed
Table 2. Serology Workup for SLE

\begin{tabular}{ll}
\hline Parameters & Test Results (Reference normal range) \\
\hline Anti-nuclear antibodies (ANA) & Strongly positive, 4+,Speckled \\
\hline Anit-ds-DNA antibody & $85 \mathrm{IU} / \mathrm{ml}$, Positive $(<30 \mathrm{IU} / \mathrm{L})$ \\
\hline Anti-Sm antibody & $62 \mathrm{IU} / \mathrm{ml}$, Positive \\
\hline Anti-Ro antibody & Negative \\
\hline Anti-La antibody & Negative \\
\hline Anti- RNP antibody & Negative \\
\hline Anti cardiolipin antibody & Negative \\
\hline Serum complement C 3 Levels & C3 - 48 mg/dL, consumed $(80-160 \mathrm{mg} / \mathrm{dL})$ \\
\hline Serum complement C 4 Levels & C4 5.8 mg/dL, consumed $(16-48 \mathrm{mg} / \mathrm{dL})$ \\
\hline Rheumatoid factor & Negative \\
\hline Anti-CCP & Negative \\
\hline C-reactive protein & Positive \\
\hline
\end{tabular}

Table 3. Investigations after 6 months treatment

\begin{tabular}{ll}
\hline Haemoglobin & Hb- $13.8 \mathrm{gm} / \mathrm{dl}$ \\
\hline Total leucocyte count & $9.030 / \mathrm{cu} \mathrm{mm}$ \\
\hline Differential leucocyte count & $\begin{array}{l}\text { Polymorphs - 65\%, Lymphocytes - 28\%, } \\
\text { Monocytes - 3\% Eosinophils-4\%, }\end{array}$ \\
\hline Platelets & $2,40,000 /$ cumm \\
\hline Erythrocyte sedimentation rate & 14 mm fall in 1st hour by Westergreen method \\
\hline Urine analysis & Albumin trace, no RBC Albumin trace, no RBC \\
\hline C-reactive protein & Normal \\
\hline Liver function tests & Serum ALT- 41U/L, Serum AST-35 U/L \\
\hline ANA & Negative \\
\hline Anti-dsDNA antibody & Negative \\
\hline Anti-Sm antibody & Negative \\
\hline C-reactive protein & Negative \\
\hline Serum Complement C 3 Levels & C3 120 mg/dL (80-160 mg/dL) \\
\hline Serum complement C 4 Levels & C4 28 mg/dL (16-48 mg/dL) \\
\hline
\end{tabular}

marked clinical improvement. and response to therapy without any recurrence or no active flare-ups of the disease so far (Tab. 3).

\section{DISCUSSION}

In 1972, KFD was first described as lymphadenitis with a focal proliferation of reticular cells, accompanied by numerous histiocytes and extensive nuclear debris occurring in young adults $<40$ years of age in Japan $[1,3,4]$. Although various infective organisms, such as Yersinia enterocolitica, Toxoplasma, Epstein-Barr virus, Cytomegalovirus, Human herpes virus, Herpes simplex virus, Hepatitis B and Parvovirus $B 19$ or autoimmune pathogenesis, have been implicated in the pathogenesis, its exact etiology is still obscure. $[1,2,3$, 4]. Clinically, it presents with non-specific symptoms such as solitary or generalized tender lymphadenopathy (100\%), mostly involving the unilateral posterior cervical group of lymph nodes $(88.5 \%)$, persistent fever $(35 \%)$, erythematous rashes $(10 \%)$, fatigue(7\%), arthralgia(7\%), arthritis (5\%), hepatosplenomegaly (3\%), night sweats and weight loss [3, 4]. The most common laboratory abnormalities include leucopenia (25-58\%), elevated ESR(40\%), anaemia (23\%) 


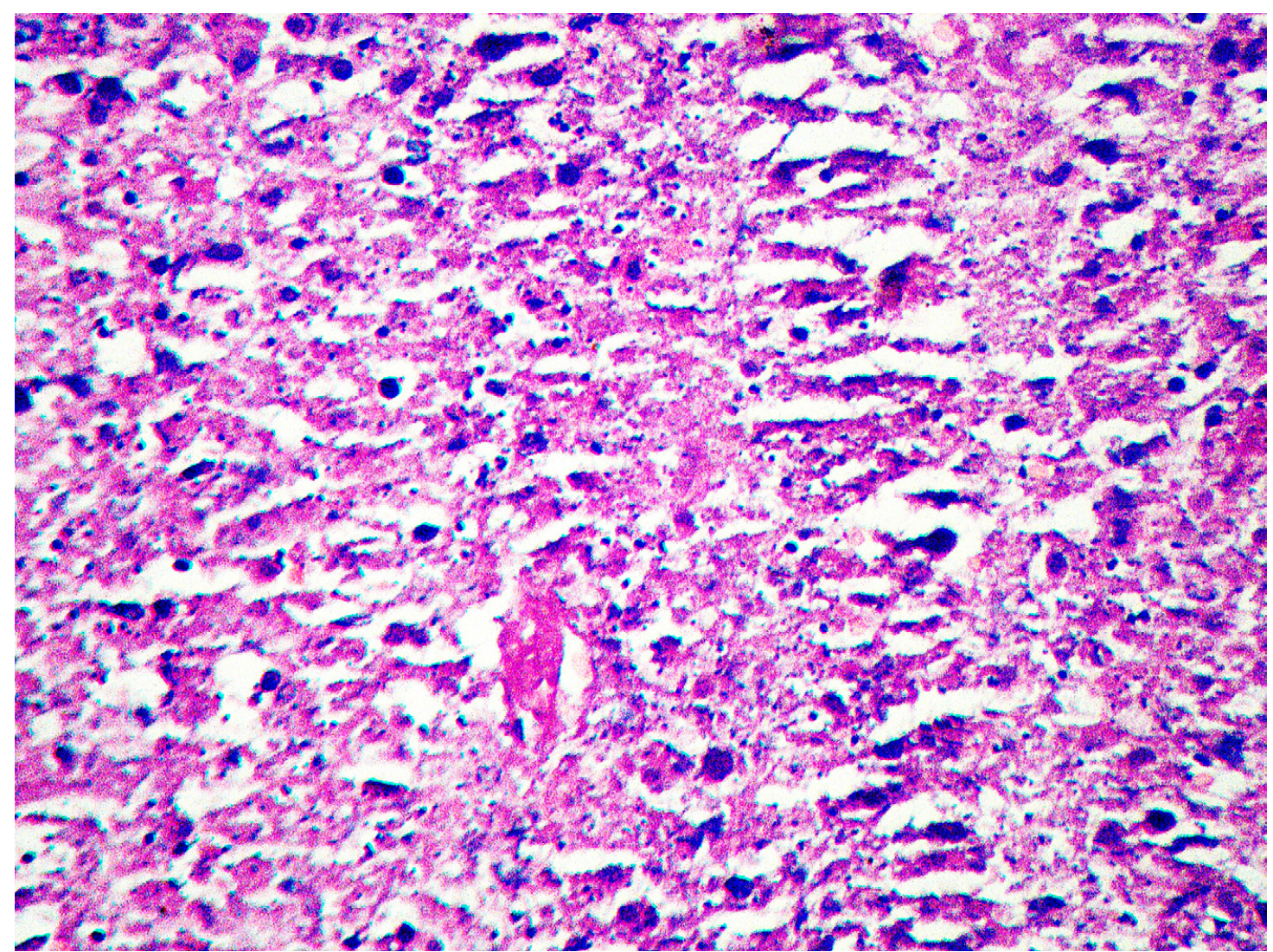

Figure 1. Fibrinoid necrosis,karyorrhectic debris with apoptotic cells. Neutrophils and eosinophils characteristically absent (haematoxylineosin stain $\times 400$ magnification).

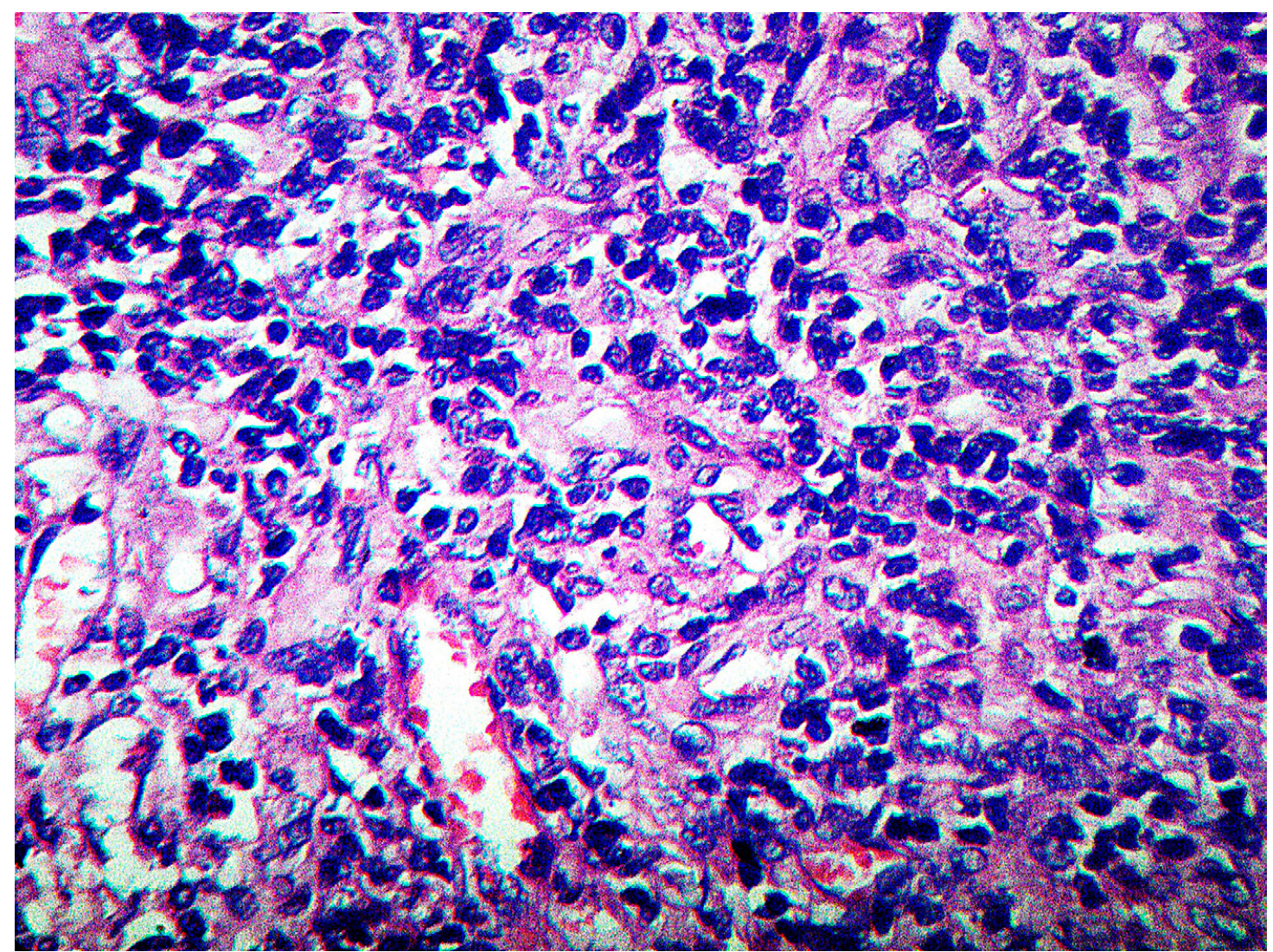

Figure 2. Numerous mature and transformed lymphocytes(immunoblasts), histiocytes, and plasmacytoid monocytes in the surrounding area of necrosis (haematoxylin-eosin stain x 400 magnification) 
atypical lymphocytes on a peripheral blood smear (25-31\%), elevated C-reactive protein (CRP), mildly elevated serum transaminases and elevated LDH $[1,4]$. Similar to KFD, SLE patients are also young females usually presenting with vague symptoms of fever, arthralgia, fatigue, and generalized lymphadenopathy. Diagnosis of SLE is confirmed by meeting minimum criteria specified by ACR, although lymphadenopathy is not among them. However, In one study of 90 SLE patients, it was observed that 23 patients (26\%) had lymphadenopathy as a presenting feature, and showed increased anti-ds DNA antibodies and decreased complement levels correlating with higher disease activity levels [5]. Usually, FNAC of the affected lymph node is strongly recommended to confirm a diagnosis in various etiology of lymphadenitis, its utility in KFD is limited with an overall diagnostic accuracy estimated at $56.3 \%$ [6]. Instead, Lymph node biopsy is preferred based on characteristic architectural and cytomorphological features revealing irregular paracortical coagulative necrotic areas made up of eosinophilic fibrinoid material interspersed with abundant karyorrhectic debris and a mixed cellular composition comprising plasmacytoid monocytes and activated T cells. No neutrophils or Eosinophils seen $[1,3]$. The area around necrotic margins shows crescentic histiocytes and thrombosed vessels [3]. Immunohistochemical (IHC) analysis of mixed cellular composition reveals a predominance of T cells, mostly CD8+ cytotoxic/suppressor subset and histiocytes, which express myeloperoxidase (MPO) and CD68 antigens [3, 4].

Kuo et al. postulated that due to differences in the cause or host reaction, KFD lymphadenitis progresses through a continuously evolving spectrum of 3 distinct histopathologic stages, Proliferative stage with a variable number of histiocytes, plasmacytoid monocytes, and karyorrhectic debris followed by Necrotizing stage with coagulative necrotic areas (Most common type) and lastly, Xanthomatous stage with a predominance of foamy histiocytes [7]. Similar to KFD, SLE can also present with necrotizing lymphadenitis. However, features that are specific to SLE-associated lymphadenitis include haematoxylin-bodies (degenerated nuclei that have reacted with antinuclear antibodies), Azzopardi phenomenon (encrustation of blood vessel walls with nuclear material), area of vasculitis surrounding the necrotic foci, and IHC finding of sparse cytotoxic CD8+ lymphocytes $[1,3]$. Due to similar symptomatology, host preferences, and pathophysiological features between KFD and SLE, a possible complex and poorly understood relationship between KFD and SLE has been postulated suggesting KFD may be a clinical feature or SLE-like autoimmune disease [2]. In one study of 244 KFD patients from 181 publications, 32 (13\%) KFD patients had SLE. Among these 32 patients, 18 (56\%) had co-existing KFD and SLE, 6(19\%) developed SLE after KFD, 4(12\%) already had SLE before KFD, and the remaining 4 (12\%) were diagnosed as incomplete SLE as they did not fulfill criteria for the definition of SLE [2]. In their study, Hu et al. observed that lymphadenitis coexisting in SLE patients, especially the necrotizing type, should be diagnosed as lupus lymphadenitis and treated accordingly. In the same study, it was also noted that 18 patients had KFD-like lymphadenitis, implying that
KFD did not always occur simultaneously with SLE [8]. In another study by Imamura et al., ultrastructural analysis of the affected lymph nodes revealed characteristic tubuloreticular structures in the lymphocytes and the endothelial cells of both SLE and KFD patients, and postulated that KFD reflects a self-limited, SLE-like autoimmune condition caused by virus-infected transformed lymphocytes [9]. However, KFD has also been reported without SLE indicating that they both are independent diseases having an autoimmune basis as it happens with most of the autoimmune diseases in susceptible subjects. From a clinical approach, SLE and KFD co-morbidity need to be stressed. KFD usually resolves in $1-4$ months with a recurrence rate of 3-4\% [3]. However, if KFD coexists with SLE, it can have a more aggressive course and may prove fatal due to severe infections, abrupt heart failure, autoimmune complications, development of haemophagocytic syndrome leading to coagulopathy and multiorgan failure $[1,2,4]$. Due to a lack of definitive markers for predicting which KFD patients will progress to SLE, it may be prudent to keep all of them on long-term clinical and serological follow-up for possible evolution of SLE.

\section{CONCLUSION}

KFD becomes an important differential diagnosis in young Asian patients with fever and cervical lymphadenopathy. Clinically, it may mimic autoimmune conditions such as SLE. Prognosis and treatment of both KFD and SLE are entirely different and if not detected early, SLE may result in multiorgan involvement with a fatal outcome. Hence, in all cases of KFD, it would be prudent to perform thorough careful histopathological examination and concomitant immunological investigations to rule out a possible association with SLE.

\section{REFERENCES}

1. Baenas DF, Diehl FA, Haye Salinas MJ, et al. Kikuchi-Fujimoto disease and systemic lupus erythematosus. Int Med Case Rep J. 2016; 9: 163-167

2. Kucukardali Y, Solmazgul E, Kunter E, et al. Kikuchi-Fujimoto disease: analysis of 244 cases. Clin Rheumatol. 2007; 26: 50-54

3. Bosch X, Guilabert A, Miquel R, et al. Enigmatic Kikuchi-Fujimoto disease: a comprehensive review. Am J Clin Pathol. 2004; 122(1): 141-52. 4. Dumas G, Prendki V, Haroche J, et al. Kikuchi-fujimoto disease. Medicine. 2014; 93(24): 372-382

5. Shapira Y, Weinberger A, Wysenbeek AJ. Lymphadenopathy in systemic lupus erythematosus. Prevalence and relation to disease manifestations. Clin Rheumatol. 1996; 15(4): 335-338.

6. Tong TR, Chan OW, Lee KC. Diagnosing Kikuchi disease on fine needle aspiration biopsy: a retrospective study of 44 cases diagnosed by cytology and 8 by histopathology. Acta Cytol. 2001; 45: 953-957.

7. Kuo TT. Kikuchi's disease (histiocytic necrotizing lymphadenitis). A clinicopathologic study of 79 cases with an analysis of histologic subtypes, immunohistology, and DNA ploidy. Am J Surg Pathol. 1995; 19: 798-809

8. Hu S, Kuo TT, Hong HS. Lupus lymphadenitis simulating Kikuchi's lymphadenitis in patients with systemic lupus erythematosus: a clinicopathological analysis of six cases and review of the literature. Pathol Int. 2003; 53: 221-226.

9. Imamura M. An ultra structural study of sub acute necrotizing lymphadenitis. Am J Pathol. 1982; 107: 292-299 